\title{
Single shot electron diffraction experiment using a sub ps MeV electron source
}

\author{
J.F. Schmerge, J. Cardoza, J. Castro, D.H. Dowell, S.M. Gierman, J.B. Hastings. H. \\ Loos, F.M. Rudakov, P.M. Weber
}

RF guns have been used for many years for injection into storage rings and to drive Free Electron Lasers (FELs). Currently photo-cathode rf guns are the brightest electron sources available for these types of high energy applications. Electrons are accelerated in rf fields greater than $100 \mathrm{MV} / \mathrm{m}$ so the beam becomes relativistic in a few cm quickly reducing the space charge force repulsion. A typical beam produced from such a gun is 5 $\mathrm{MeV}$ with sub ps pulse length and greater than $10^{8}$ electrons per bunch. The relatively high beam energy requires several meter drift distance between target and detector but allows single shot diffraction images to be captured with sub ps resolution. The first results from a diffraction experiment using a $160 \mathrm{~nm}$ thick Al target will be presented along with the measured gun beam parameters. 